\title{
OPINION DYNAMICS: KINETIC MODELLING WITH MASS MEDIA, APPLICATION TO THE SCOTTISH INDEPENDENCE REFERENDUM
}

\author{
LAURENT BOUDIN AND FRANCESCO SALVARANI
}

\begin{abstract}
We consider a kinetic model describing some mechanisms of opinion formation in the framework of referendums, by allowing that the individuals, who can interact between themselves and modify their opinion by means of spontaneous self-thinking, are moreover under the influence of mass media. After proving the main properties of the model, such as existence of solutions and conservation properties, we study, at the numerical level, both the transient and the asymptotic regimes. In particular, we point out that a plurality of media, with different orientations, is a key ingredient to allow pluralism and prevent consensus. The forecasts of the model are compared to some polls related to the Scottish independence referendum of 2014.
\end{abstract}

\section{INTRODUCTION}

Forecasting the opinion evolution with respect to a binary question is crucial in many situations. A typical example consists in the anticipation of a referendum results of competition by using poll data from surveys held a few weeks before the vote. Our work lies in the sociophysics domain, which was born in the 80s. The reader can check [10] and the references therein to know more about the topic. In particular, one can find there some discussions about the French referendum on the European constitution in 2005.

In this article, we give a contribution to this problem by studying a mathematical model based on a kinetic approach. We perform the following hypotheses.

Assumption 1. The number of individuals in the population is constant. Of course, this is only relevant for short-term forecasts, as during a referendum campaign.

Assumption 2. The community is fully interconnected and all the members can somehow discuss with each other. By tuning the cross section

This work was partially funded by the 2013-2014 French CNRS PEPS HuMaIn project Kindymo, headed by L. Boudin, the French ANR-13-BS01-0004 project, Kibord, headed by L. Desvillettes, and the Italian national institute of higher mathematics (INDAM), GNFM project Kinetic description of collective phenomena: theory and numerical simulations, headed by F. Salvarani. 
of the model, however, it is possible to generalize our model to situations where the interaction probability between individuals depends on their own convictions.

Assumption 3. There exists a number of mass media which can have an effect on the individuals. This assumption is natural in most countries, since media are both observers and opinion carriers.

Let us more focus on the mass media, whose involvement is the most significant contribution of this work. Although the press freedom appears as a safeguard in a democracy [14], the media can also be used to influence the public opinion, by taking advantage of their possibility of easily reaching a wide audience and favour some interests, ideas or arguments inside a population [7]. In order to influence the population, many different tactics have been developed. Among them, we quote the distraction, in different forms (which is based on the assumption that the public has a limited attention span), the appeal to consensus and the fear mongering [9]. These techniques are used either separately or combined between them, and could be applied to many different contexts. However, they need some common factors in order to be successful.

Mass media use diversified media technologies in order to reach a large audience and transfer concepts, ideas, etc. Among them, we can cite broadcast media (radio, television), print media (newspapers, books), outdoor media (posters), digital media (Internet, mobile mass communication).

However, mass media often simply spread out the opinions which they support into the population, without any other interest than the maximisation of their audience.

These two different strategies cannot be modelled in the same way. Manipulating media can be described as entities with a given external opinion, which aim to spread their own opinion inside the population. Wide audience media should be described with a more intricate approach: the opinions they carry can depend on the opinion distribution inside the population. Hence, in this latter case, the influence of the population on the media opinion has to be part of the model itself.

In this article, we only consider media with an a priori given opinion. Note that this case can be seen as a first approximation of the wide audience media, where the media opinion may not remain constant, but does not depend on the opinion distribution.

We here use a kinetic approach to describe and forecast the evolution of a system under the aforementioned effects. In this framework, the information on the system is contained in a distribution function whose time evolution is governed by a partial differential equation with integral operators. This strategy is based on sophisticated mathematical tools and its interest is apparent when the number of individuals is very large, since it allows to handle a collective behaviour. 
Kinetic equations have been used to model social phenomena since the early 90s, when Helbing studied behavioural changes by using Boltzmannlike equations $[12,11,13]$. Subsequently, this approach has been the basis of several works, see the review article [6] and the references therein. Note that, in most part of the literature based on the kinetic approach, the key phenomenon is compromise (see, for example, $[1,2,15,8]$ ).

The model we here investigate also owns this binary interaction feature, by supposing that individuals follow the rule proposed by the authors in [5]. By taking account the influence of mass media on the population, we are able to improve our previous results in [5] and recover some realistic behaviours.

The opinion variable of our model runs in a continuous way between two extreme values, from -1 to +1 . The model describes the time evolution of a distribution function $f$ (depending on both opinion and time), which represents the density of individuals having an opinion about the binary question. It reflects the opinion formation process, which is not necessarily of binary type, since the doubt and the partial agreement are often predominant feelings.

The media action on the system is modelled by a kinetic operator which has a structure similar to the media operator introduced in [4], but with a different nonlinear effect in the post-interaction opinion. The structure of this linear term (where the linearity is intended only with respect to $f$ ) is well adapted to the coupling with the self-thinking.

At the end of the opinion formation process, the opinions must be translated into a decision. This second step is not tackled here, since it is often the result (at least in the case of reasonable and rational individuals) of a game-theoretical approach, especially when those individuals have intermediate opinions.

Since we are working with a continuous opinion variable, it is difficult, in general, to get comparisons with real data. Indeed, polls usually have a binary or ternary structure, since they are interested in forecasting the result of the final choice with respect to the binary question of the referendum. However, some more structured surveys have been built on a more complete scale (usually from 1 to 10). These polls can be a good tool for comparisons, which are essential to somehow validate our model. The last part of our study is precisely devoted to the qualitative comparison between the results of our model and three surveys performed by the polling corporation ICM Unlimited $^{1}$ about the Scottish independence referendum, that took place in Scotland on September 18, 2014.

The article is organized as follows: in the next section, we describe the model and its mathematical structure. The existence of solutions is investigated in Section 3, whereas the numerical simulations are performed and

\footnotetext{
${ }^{1}$ See http://www.icmunlimited.com/media-centre/polls/
} 
commented in Section 4. Eventually, Section 5 focuses on the Scottish independence referendum: we relate our numerical results to some existing polls obtained before the referendum itself. It provides some kind of validation to our model, and already suggests possible improvements.

\section{KinetiC MODEL}

In this section, we briefly describe the model we investigate here. As already stated, it appears as an extension to the one previously introduced in [5]. We refer to that article for a full description of the bilinear integral operator defining the binary interaction between the agents.

In what remains, $\Omega$ denotes the open interval $(-1,1)$. The variables of the model are opinion $x$, a continuous variable belonging to $\bar{\Omega}$, and time $t \in \mathbb{R}^{+}$. The opinion variable $x$ describes the degree of agreement with respect to a binary question (for example, a referendum). In particular, $x= \pm 1$ are the two extreme answers to the question, i.e. "yes" or "no" without reserve, whereas any intermediate value between -1 and $+1,0$ excluded, means that the corresponding agent partially agrees with the opinion labelled with the same sign, with a degree of conviction which is proportional to $|x|$. The value $x=0$ means that the agent is undecided.

The community is described by means of the distribution function $f:=$ $f(t, x)$, defined on $\mathbb{R}_{+} \times \bar{\Omega}$, whose time evolution is governed by a kinetic equation. The main ingredients of the kinetic model act on two different levels. The first one is the description of the microscopic active phenomena, in this case the self-thinking, the binary opinion exchange between the individuals of the population and the effect of mass media on the single individual. The second level governs the time evolution of the distribution function, which is induced by the operators which take the microscopic phenomena into account. Since the model is of kinetic type, we borrow the language of kinetic theory. For instance, the term collision means an interaction with exchange of opinions, that gives, as a result, a modification of the agents opinions.

2.1. Self-thinking. The self-thinking phenomenon is described by a diffusion operator obeying to a non-uniform Fourier law, with Fourier term $\alpha=\alpha(x)$. This term quantifies the possibility that people may change their opinion through personal reasoning. In particular, we assume that the Fourier term satisfies the following properties.

Definition 2.1. Let $\alpha: \bar{\Omega} \rightarrow \mathbb{R}$ be a nonnegative function of class $C^{1}(\bar{\Omega})$. We say that $\alpha$ is admissible if

- $\alpha(x)=\alpha(-x)$ for any $x \in \bar{\Omega}$,

- $\alpha(-1)=\alpha(+1)=0$.

2.2. Binary opinion exchanges. Let $x, x_{*} \in \bar{\Omega}$ the opinions of two individuals of the population before interacting, and $x^{\prime}, x_{*}^{\prime} \in \bar{\Omega}$ the corresponding opinion after the binary exchange. 
We suppose that the individuals of the population are of conciliatory type $[5,3]$ : it means that they have a natural trend to reach a compromise with each other. From a quantitative point of view, the opinions after interaction are modified in order to get closer to the average opinion before the interaction and, at the same time, stronger opinions are less attracted towards the average than weaker ones. More precisely, the collision rule adopted in the article is the following:

$$
x^{\prime}=\frac{x+x_{*}}{2}+\eta(x) \frac{x-x_{*}}{2}, \quad x_{*}^{\prime}=\frac{x+x_{*}}{2}+\eta\left(x_{*}\right) \frac{x_{*}-x}{2} .
$$

The function $\eta: \bar{\Omega} \rightarrow \mathbb{R}$ is called the attraction function, and satisfies the following properties.

Definition 2.2. A function $\eta \in C^{1}(\bar{\Omega})$ is admissible if $0 \leq \eta<1, \eta^{\prime}(x)>0$ for all $x>0$, and the Jacobian $J\left(x, x_{*}\right)$ of the collision mechanism (1) is lower bounded by a positive constant, i.e. there exists $J_{0}>0$ such that $J\left(x, x_{*}\right) \geq J_{0}$ for any $x, x_{*} \in \Omega$.

The collision rule (1) governs the interaction between individuals and the corresponding exchange of opinions, through a collisional integral of Boltzmann type.

2.3. Interaction with mass media. In the model, we take into account the existence of $m$ mass media, $m \geq 1$. They can influence the population by sharing their opinion about the referendum. The effects of the media are modelled thanks to an interaction with a given background. To define the characteristics of each media $M_{j}, 1 \leq j \leq m$, we need three quantities: its time-depending strength $\theta_{j}: \mathbb{R}^{+} \rightarrow \mathbb{R}^{+}$, its time-depending opinion $X_{j}: \mathbb{R}^{+} \rightarrow \bar{\Omega}$, and its attractiveness $q_{j}: \bar{\Omega}^{2} \rightarrow \mathbb{R}$.

The individuals of the population are still considered as conciliatory regarding the media. The evolution of an individual's opinion follows a rule, similar to the binary collision mechanism defined in the previous subsection, but with the difference that the opinion of the media is not influenced by the opinion of the population. The main idea of the collision mechanism consists in supposing that the opinions after the interaction will be modified in order to approach the average opinion before the interaction and, again, that stronger opinions are less attracted towards the media opinion than weaker ones. Hence, if we denote ny $\bar{x}_{j}$ the post-collisional opinion of an individual of pre-collisional opinion $x$, for each mass media $M_{j}, j \in[1, \ldots, m]$, we write

$$
\bar{x}_{j}=\frac{x+X_{j}(t)}{2}+q_{j}\left(x, X_{j}(t)\right) \frac{x-X_{j}(t)}{2},
$$

where $q_{j}$ satisfies the following properties.

Definition 2.3. For any $j$, the media attractiveness $q_{j} \in C^{0}(\bar{\Omega} \times \bar{\Omega})$ is admissible if $0 \leq q_{j} \leq 1$ for any $x \in \bar{\Omega}$. 
Moreover, it seems reasonable, from the modelling point of view, to impose that the media attractiveness equals 1 when $|x| \simeq 1$ : it means that individuals with a strong opinion are less influenced by the media effects than agents with a weaker opinion.

2.4. Time evolution of $f$. The model governing the evolution of the system is a partial differential integral equation, which can be more naturally written in a weak form. Indeed, the existence of a pre-collisional opinion pair generating a post-collisional opinion pair $\left(x, x_{*}\right)$ through the collision rule (1) is not guaranteed, in general. The model hence reads as follows. Let $T>0$ and $f^{\text {in }} \in L^{1}(\Omega)$ a nonnegative function. The unknown $f$ satisfies, for almost every $t \in[0, T]$ and in a distributional sense in $t$,

$$
\frac{\mathrm{d}}{\mathrm{d} t}\left(\int_{\Omega} f \varphi \mathrm{d} x\right)=\int_{\Omega} \partial_{x}\left(\alpha \partial_{x} \varphi\right) f \mathrm{~d} x+\langle Q(f, f), \varphi\rangle+\sum_{j=1}^{m}\left\langle L_{j}(f), \varphi\right\rangle
$$

for any test-function $\varphi \in C^{2}(\bar{\Omega})$, with initial condition

$$
f(0, x)=f^{\text {in }}(x), \quad x \in \bar{\Omega} .
$$

Operator $Q$ translates the effects of the binary interactions between the agents, and can be written in different forms. A key ingredient of this bilinear (with respect to $f$ ) operator is the cross-section. This nonnegative quantity, denoted by $\beta$, governs the probability that an exchange of opinions can occur. We here assume that $\beta$ is a positive constant. This is the simplest possible assumption. It implies that the probability of interaction between two individuals depends neither on their respective opinions, nor on time, and that there are no forbidden exchanges.

The weak form used in this work is

$$
\langle Q(f, f), \varphi\rangle=\beta \iint_{\Omega^{2}} f(t, x) f\left(t, x_{*}\right)\left[\varphi\left(x^{\prime}\right)-\varphi(x)\right] \mathrm{d} x_{*} \mathrm{~d} x .
$$

Note that the collision rule (1) then only appears in the arguments of the test-functions.

Operators $L_{j}, 1 \leq j \leq m$, describe the media effect on the population, and they have the following form:

$$
\left\langle L_{j} f, \varphi\right\rangle=\theta_{j}(t) \int_{\Omega} f(x)\left(\varphi\left(\bar{x}_{j}\right)-\varphi(x)\right) \mathrm{d} x .
$$

Note that those operators linearly depend on $f$.

\section{Main mathematical Properties}

It is easy to deduce some basic mathematical properties, such as the conservation of the number of individuals in the population.

Proposition 3.1. Let $f=f(t, x)$ be a nonnegative weak solution of (3)-(4), with a nonnegative initial datum $f^{\text {in }} \in L^{1}(\Omega)$. Then we have

$$
\|f(t, \cdot)\|_{L^{1}(\Omega)}=\left\|f^{\mathrm{in}}\right\|_{L^{1}(\Omega)} \quad \text { for a.e. } t \geq 0 .
$$


Proof. This is a straightforward consequence of (3)-(6), with test-function $\varphi \equiv 1$.

Some mathematical properties of the equation are a consequence of the structure of the bilinear term. The following result holds, the proof of which is given in [5].

Lemma 3.2. Let $f(t, \cdot) \in L^{1}(\Omega)$. Then both $Q^{+}(f, f)(t, \cdot)$ and $Q(f, f)(t, \cdot)$ belong to $L^{1}(\Omega)$, and we have, for a.e. $t>0$,

$$
\begin{aligned}
\left\|Q^{+}(f, f)(t, \cdot)\right\|_{L^{1}(\Omega)} & \leq \frac{2 \beta}{1-\max \eta}\|f(t, \cdot)\|_{L^{1}(\Omega)}^{2}, \\
\|Q(f, f)(t, \cdot)\|_{L^{1}(\Omega)} & \leq\left(\frac{2}{1-\max \eta}+1\right) \beta\|f(t, \cdot)\|_{L^{1}(\Omega)}^{2} .
\end{aligned}
$$

Existence of a nonnegative solution to (3)-(4) can be deduced by construction, starting from the following result, again proven in [5].

Proposition 3.3. Consider the initial-boundary value problem for the unknown $v=v(t, x), x \in \Omega$ and $t \in[0, T]$,

$$
v_{t}-\left[\alpha(x) v_{x}\right]_{x}+\mu v=g, \quad \mu \geq 0,
$$

with initial condition

$$
v(0, \cdot)=v^{\text {in }}
$$

and boundary conditions

$$
\lim _{x \rightarrow \pm 1} \alpha(x) v_{x}(t, x)=0 \quad \text { a.e. } t,
$$

where $v^{\text {in }} \in L^{1}(\Omega), g \in C\left([0, T] ; L^{1}(\Omega)\right)$ are nonnegative functions. Then (9)-(11) admits a unique solution $v \in C^{0}\left([0, T] ; L^{1}(\Omega)\right)$, and $v$ is nonnegative.

The procedure to build a nonnegative weak solution is based on the monotonicity properties of our problem.

Theorem 3.4. Let $f^{\text {in }}$ a nonnegative function in $L^{1}(\Omega)$. Then there exists a nonnegative weak solution $f \in L^{\infty}\left(0, T ; L^{1}(\Omega)\right)$ to $(3)-(4)$.

Proof. Set

$$
\rho=\int_{\Omega} f^{\text {in }}\left(x_{*}\right) \mathrm{d} x_{*} .
$$

Consider the sequence $\left(f^{n}\right)_{n \in \mathbb{N}}$ of functions, defined as solutions to

$$
\int_{\Omega} \partial_{t} f^{n+1} \varphi \mathrm{d} x-\int_{\Omega} \partial_{x}\left(\alpha \partial_{x} \varphi\right) f^{n+1} \mathrm{~d} x
$$

$+\left[\beta \rho+\sum_{j} \theta_{j}(t)\right] \int_{\Omega} f^{n+1} \varphi \mathrm{d} x=\left\langle Q^{+}\left(f^{n}, f^{n}\right), \varphi\right\rangle+\sum_{j} \theta_{j}(t) \int_{\Omega} f^{n} \varphi\left(\bar{x}_{j}\right) \mathrm{d} x$, 
with $f^{0} \equiv 0$, for all $\varphi \in C^{2}(\bar{\Omega})$, satisfying the initial and boundary conditions

$$
f^{n}(0, \cdot)=f^{\text {in }}, \quad n \geq 1, \quad \lim _{x \rightarrow \pm 1} \alpha(x) f_{x}^{n}(t, x)=0 \text { for a.e. } t, n \geq 1 .
$$

Thanks to Lemma 3.2, we can apply Proposition 3.3 and deduce, by induction, that $f^{n}$ exists, belongs to $C^{0}\left([0, T] ; L^{1}(\Omega)\right)$ and is nonnegative.

Then, choosing $\varphi=1$ in (12), we obtain

$$
\begin{aligned}
\frac{\mathrm{d}}{\mathrm{d} t} \int_{\Omega} f^{n+1} \mathrm{~d} x+\left[\beta \rho+\sum_{j} \theta_{j}(t)\right] & \int_{\Omega} f^{n+1} \mathrm{~d} x \\
& =\beta\left(\int_{\Omega} f^{n} \mathrm{~d} x\right)^{2}+\sum_{j} \theta_{j}(t) \int_{\Omega} f^{n} \mathrm{~d} x .
\end{aligned}
$$

Therefore, by finite induction, we immediately get

$$
\int_{\Omega} f^{n} \mathrm{~d} x \leq \rho, \quad n \geq 1 .
$$

Moreover, by applying the same strategy as in [5], we can prove that $\left(f^{n}\right)$ is a non-decreasing sequence.

By monotone convergence, we finally deduce the existence of $f$ as the limit of $\left(f^{n}\right)$ in $L^{\infty}\left(0, T ; L^{1}(\Omega)\right)$. In order to check that $f$ satisfies (3), we write

$$
\begin{gathered}
\int_{0}^{T} \int_{\Omega} f^{n+1} \varphi(x) \psi(t) \mathrm{d} x \mathrm{~d} t-\int_{0}^{T} \int_{\Omega}\left(\alpha(x) \varphi^{\prime}(x)\right)^{\prime} f^{n+1} \psi(t) \mathrm{d} x \mathrm{~d} t \\
+\left(\beta \rho+\sum_{j} \theta_{j}(t)\right) \int_{0}^{T} \int_{\Omega} f^{n+1} \varphi(x) \psi(t) \mathrm{d} x \mathrm{~d} t \\
=\beta \int_{0}^{T} \iint_{\Omega^{2}} f^{n}(t, x) f^{n}\left(t, x_{*}\right) \varphi\left(x^{\prime}\right) \psi(t) \mathrm{d} x \mathrm{~d} x_{*} \mathrm{~d} t \\
+\sum_{j} \int_{0}^{T} \int_{\Omega} \theta_{j}(t) f^{n} \varphi\left(\bar{x}_{j}\right) \psi(t) \mathrm{d} x \mathrm{~d} t .
\end{gathered}
$$

The only small difficulty lies in the fourth integral. It also converges since $\iint_{\Omega^{2}}\left|f^{n}(x) f^{n}\left(x_{*}\right)-f(x) f\left(x_{*}\right)\right|\left|\varphi\left(x^{\prime}\right)\right| \mathrm{d} x \mathrm{~d} x_{*} \leq 2 \rho\left\|f^{n}(t, \cdot)-f(t, \cdot)\right\|_{L^{1}}\|\varphi\|_{L^{\infty}}$.

That ends the proof of Theorem 3.4.

\section{Numerical tests}

The architecture of the numerical method used in the simulations treats separately the kinetic part and the diffusion term by means of a splitting technique. More details about the numerical scheme are given in [5], except for the media numerical treatment. The mass media are a set of invariant particles which carry the media opinion and interact with the distribution 
function $f$, chosen by a sample over the whole set of macro-particles, by obeying to the collision rules (2).

In all the computations, the Fourier term is $\alpha(x)=0.05\left(1-x^{2}\right)^{1 / 3}$, which is in agreement with the hypotheses of Definition 2.1, the collision frequency $\beta$ is set to 50 , and the attraction function is given by $\eta(x)=0.25\left(1+x^{2}\right)$, which satisfies the constraints of Definition 2.2.

The media attractivenesses $q_{j}$ are crucial. In our tests, we shall consider two different types of media influence: a global media influence function and a media influence which equals 1 outside an interval $[-\delta, \delta], 0<\delta<1$. This last choice translates the idea that strong opinions are not affected by the influence of the media, whereas weaker opinions are sensitive to the effect of mass media.

In what follows, we investigate some relevant test cases, which give an overview of the quantitative features of the model. First of all, we study the influence on a population of a unique media with a fixed opinion, and two different initial conditions: a balanced initial datum and an unbalanced one. Subsequently, we discuss the interactions between two groups of balanced media, again with fixed opinions. This last result is compared in Section 5 to the opinion dynamics for the Scottish independence referendum.

4.1. Effect of a unique media on the opinion formation. The first example studies the behaviour of the initial value problem (3)-(4), with $m=1$. In all tests of this subsection, we suppose that $\theta_{1}$ is constant equal to 50 .

The media attractiveness is also constant: $q_{1}=0.5$. In Figure 1 , we can check that the asymptotic states of the system with media opinion $X_{1}(t)=$ -0.9 , for both initial data $f_{1}^{\text {in }}=0.5$ (plot on the left) and $f_{2}^{\text {in }}=\mathbf{1}_{[0,1]}$ (plot on the right).

We note that the asymptotic states are the same, and of course do not depend on initial data. They are driven by the media opinion, even if the population has an initial opinion with opposite sign with respect to the media opinion. Moreover, both situations show that self-thinking prevents the system from a complete adherence to the media opinion.

In Figure 2, we can see the sensitivity of the model with respect to the media attractiveness $q_{1}$. We here choose a regularized version of

$$
q_{1}=1-\frac{1}{2} \mathbf{1}_{[-0.5,0.5]} \text {. }
$$

Note that, since we are working on a discrete grid, we can numerically identify the discretization of $q_{1}$ and the discretization of its regularized version.

This media attractiveness means that only individuals with a weak opinion, in the range $[-0.5,0.5]$, are influenced by the media opinion.

In order to compare the results with the previous case, we choose the same set of initial datum and media opinion as in the second example, i.e. $f_{2}^{\text {in }}=\mathbf{1}_{[0,1]}$ and $X_{1}(t)=-0.9$. 

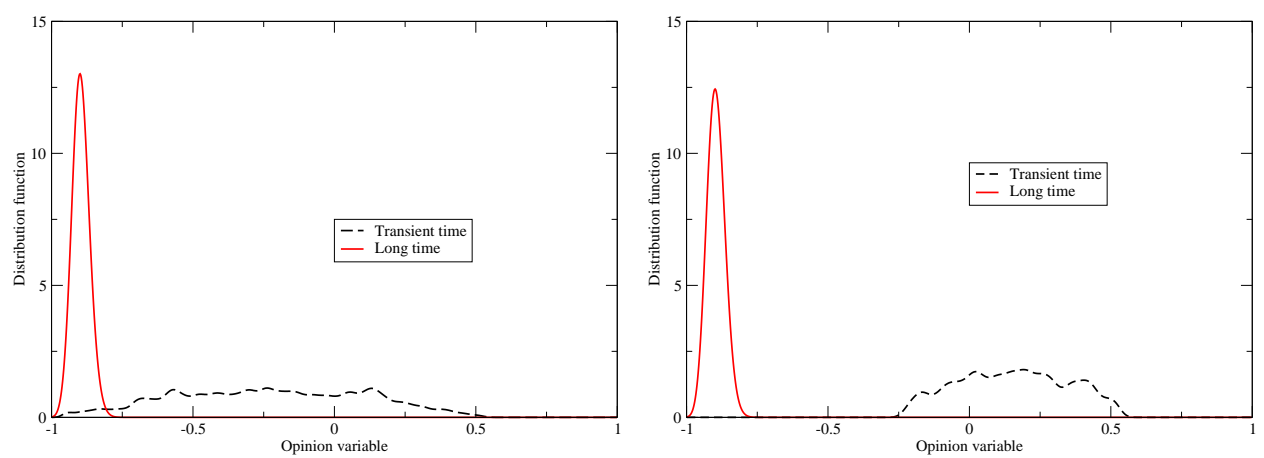

Figure 1. Effect of a unique media for two different initial data.

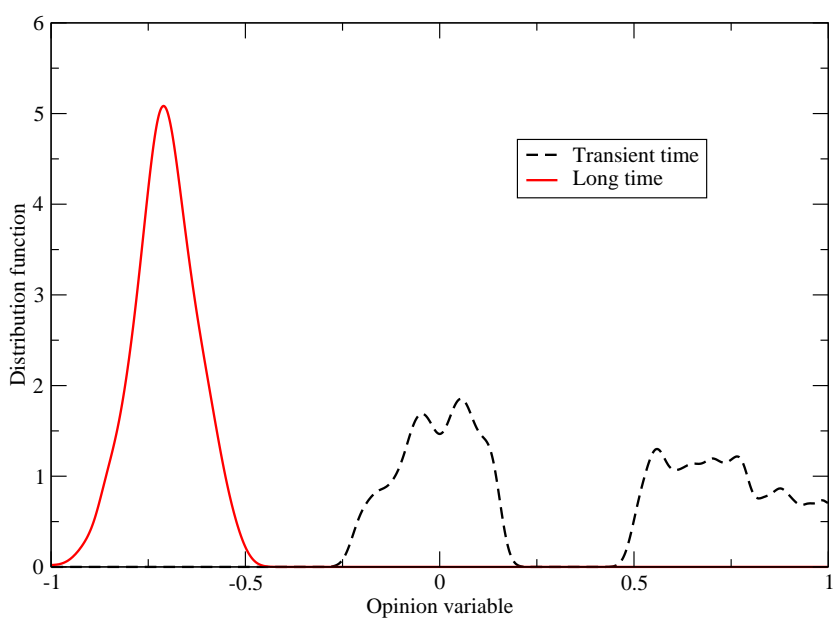

FiguRE 2. Effect of a unique media with finite influence range.

After a transient period of time, the media has, again, enough strength to drive the whole population towards opinions whose sign agrees with its opinion, even if $50 \%$ of the population cannot initially be influenced by the media opinion.

This behaviour is due to the binary interactions between the agents. Since the whole population is composed of conciliatory individuals, the effect of the binary interactions leads to compromise. Hence, the fraction of the population which keeps an opinion outside $(0.5,1]$ decreases towards 0 . On the other hand, since the agents with opinions inside the interval $[-1,-0.5)$ are not influenced by the media, the peak of the distribution is not centred at the media opinion any more, and is closer to 0.

4.2. Interaction between two media with fixed opinions. We conclude by pointing out how a plurality of media is a factor that allows pluralism of opinions and the splitting of the population in two well-defined groups. 
In order to recover this behaviour, we now choose $m=2, \theta_{1}=\theta_{2}=50$, and $X_{1}(t)=-X_{2}(t)=0.9$.

In Figure 3, we plot the asymptotic state of the distribution function, with initial datum $f_{1}^{\text {in }}=0.5$ and both media attractivenesses given by $q_{j}=1$ if $\left|x-X_{j}\right|>1$ and $q_{j}=0.5$ otherwise, for $j=1,2$.

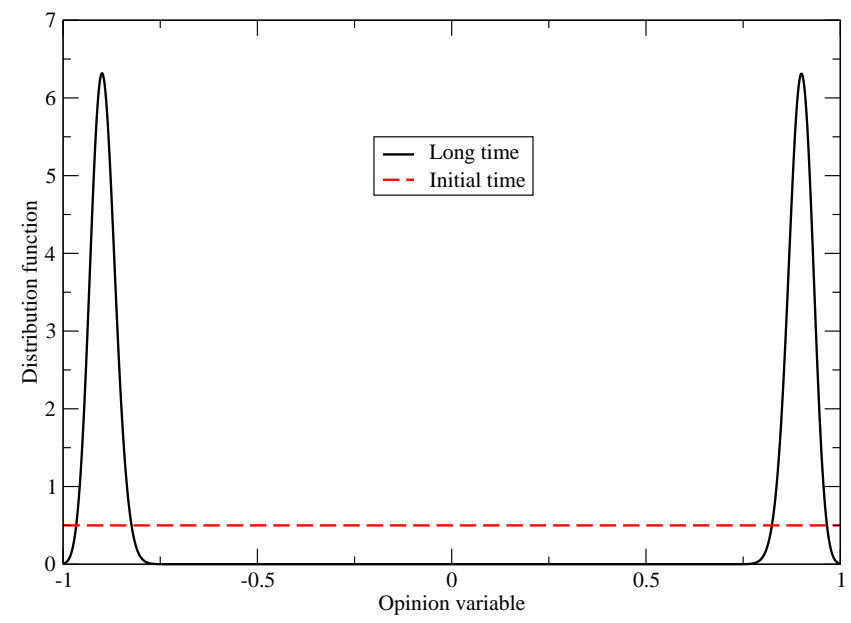

Figure 3. Pluralism of media and pluralism of opinions: global media influence

After a transient period, we note the formation of two peaks centred at each media opinion \pm 0.9 . The fraction of agents with a weak opinion is very low. It is a straightforward consequence of the fact that all individuals of the population are under the media influence.

In Figure 4, we plot the asymptotic state of the distribution function, with the same initial datum as in Figure 3 , namely $f_{1}^{\text {in }}=1 / 2$. The media attractivenesses are given by $q_{j}=1$ if $\left|x-X_{j}\right|>0.5$ and $q_{j}=0.5$ otherwise, for $j=1,2$.

In this case, the individuals with opinion close to 0 are not influenced by any media: they can change their opinions only through self-thinking and binary interactions.

We note the formation of two strong sub-groups with asserted opinions, but also the emergence of a fraction of undecided persons (who may have a higher propensity to abstention). This behaviour is typical of some referendum campaigns, such as the case discussed in the next section.

\section{Comparison with polls: the Scottish Case}

On September 18, 2014, Scotland was called to answer the independence referendum question: "Should Scotland be an independent country?". The turnout of $84.6 \%$ was the highest one recorded for a referendum in the United Kingdom since the introduction of universal suffrage. This datum is enough, by itself, to prove the importance that Scotland attributed to the 


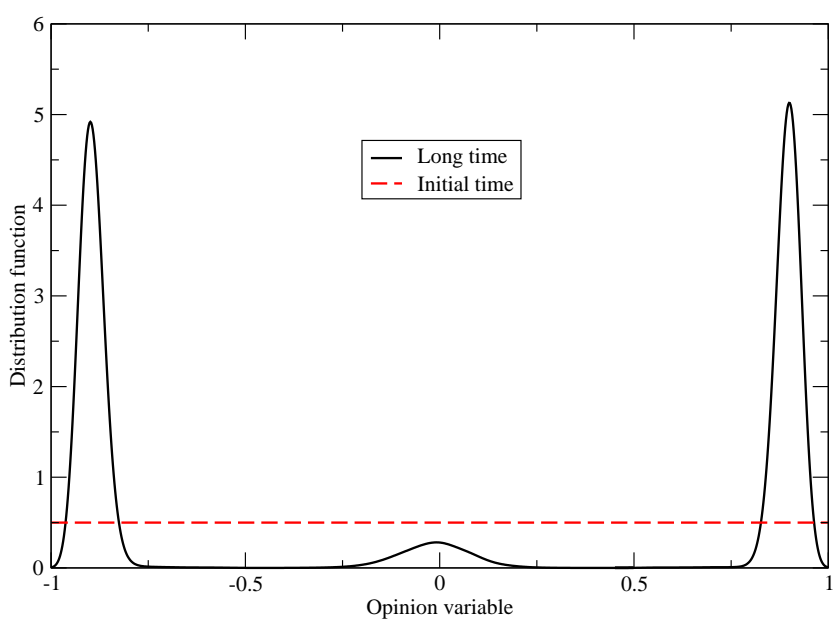

Figure 4. Pluralism of media and pluralism of opinions: local media influence.

referendum question. The official results, certified by the Chief Counting Officer, from the Electoral Management Board for Scotland (EMB), are the following:

\begin{tabular}{|l|r|r|} 
Yes & $1,617,989$ & $44.7 \%$ \\
No & $2,001,926$ & $55.3 \%$ \\
Valid votes & $3,619,915$ & $99.91 \%$ \\
Invalid or blank votes & 3,429 & $0.09 \%$ \\
Total votes & $3,623,344$ & $100.00 \%$
\end{tabular}

The referendum was preceded by hundreds of surveys, whose aim was the prediction of the final result. Those surveys took into consideration many different aspects related to the independence referendum question. Among them, we have considered three surveys for the validation phase, which have the advantage to offer multiple answers and allow the comparison with our model, based on a continuum of opinions. The precise data of these polls are the following:

(1) July 11, 2014: ICM survey for Scotland on Sunday with 1002 respondents, aged 16 and older;

(2) August 13, 2014: ICM survey for Scotland on Sunday with 1005 respondents, aged 16 and older;

(3) September 16, 2014: ICM survey for The Scotsman with 1175 respondents, aged 16 and older.

The question was identical in the three polls: "Can you please say where you are on this scale regarding Scotland becoming independent?". The answers were modulated on a scale, between 1 and 10, where

- 1 means "completely against an independent Scotland",

- 5 means "neither for nor against" (positioned between 5 and 6), 
- 10 means "completely for an independent Scotland".

The results of the three surveys are all plotted in Figure 5.

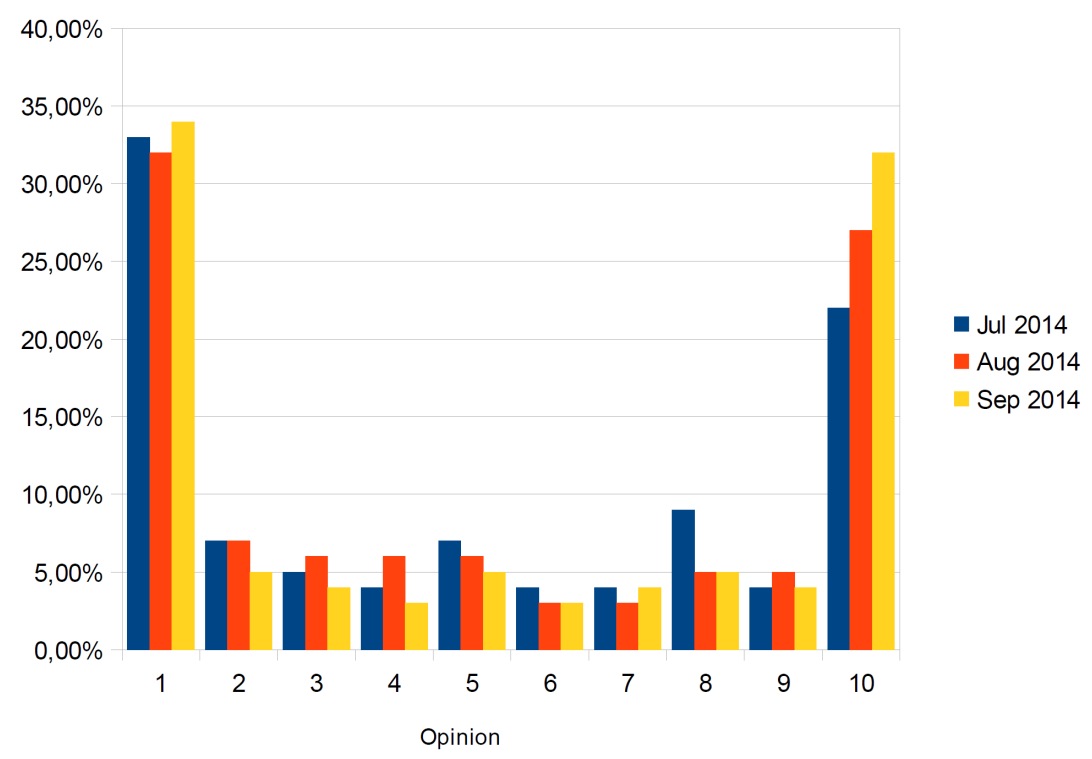

FiguRE 5. Results of the three ICM surveys.

As the referendum day came, there was a clear bipolarization of the population and the emergence of two opposite fields with approximately the same magnitude. The number of undecided and agents with mild opinion decreased, and the media widely spread the arguments of both opposite parties.

The qualitative agreement between the surveys history and the results in Figure 4 is quite good: both peaks are centred at the extreme values (or very close to them). We recognize moreover the existence of some undecided people, which have been reported in the polls (there is a local maximum around the value 5).

The main differences between our simulations and the three surveys is given by the behaviour of moderately decided individuals (located in states 2 to 4 and 7 to 9 ). Indeed, the polls report that a small, but not negligible, fraction of polled people is located in these intermediary states, whereas, in our numerical simulation, the fraction of the population far from the peaks is very small. However, it is difficult to draw some conclusions from this consideration since the magnitude of the population in each of the intermediary states is often below $5 \%$, and therefore it is quite close to the survey error margin. 


\section{Conclusion}

We presented a kinetic model to describe an interconnected population which must choose an option in the framework of a binary question. The agents can modify their opinion by means of spontaneous self-thinking, discussions with each other, and influence of mass media. The model has been investigated both mathematically and numerically. The comparison between the model and the results of three surveys on the Scottish independence referendum of 2014 provides a good qualitative agreement.

\section{REFERENCES}

[1] E. Ben-Naim, P. L. Krapivsky, and S. Redner. Bifurcation and patterns in compromise processes. Physica D, 183(3-4):190-204, 2003.

[2] E. Ben-Naim, P. L. Krapivsky, F. Vazquez, and S. Redner. Unity and discord in opinion dynamics. Physica A, 330(1-2):99-106, 2003. Randomness and complexity (Eilat, 2003).

[3] L. Boudin, A. Mercier, and F. Salvarani. Conciliatory and contradictory dynamics in opinion formation. Physica A, 391(22):5672-5684, 2012.

[4] L. Boudin, R. Monaco, and F. Salvarani. Kinetic model for multidimensional opinion formation. Phys. Rev. E (3), 81(3):036109, 9, 2010.

[5] L. Boudin and F. Salvarani. A kinetic approach to the study of opinion formation. M2AN Math. Model. Numer. Anal., 43(3):507-522, 2009.

[6] L. Boudin and F. Salvarani. Modelling opinion formation by means of kinetic equations. In Mathematical modeling of collective behavior in socio-economic and life sciences, Model. Simul. Sci. Eng. Technol., pages 245-270. Birkhäuser Boston Inc., Boston, MA, 2010.

[7] M. Coxall. Human manipulation, a handbook. Malcolm Coxall, Cornelio Books, 2013.

[8] B. Düring, P. Markowich, J.-F. Pietschmann, and M.-T. Wolfram. Boltzmann and Fokker-Planck equations modelling opinion formation in the presence of strong leaders. Proc. R. Soc. A, 465(2112):2687-3708, 2009.

[9] J. Ellul. Propaganda: the formation of men's attitudes. Vintage Books, New York, 1973.

[10] S. Galam. Sociophysics: a physicist's modeling of psycho-political phenomena (understanding complex systems). Springer, 2012.

[11] D. Helbing. Boltzmann-like and Boltzmann-Fokker-Planck equations as a foundation of behavioral models. Physica A, 196:546-573, 1993.

[12] D. Helbing. Stochastic and Boltzmann-like models for behavioral changes, and their relation to game theory. Physica A, 193:241-258, 1993.

[13] D. Helbing. A mathematical model for the behavior of individuals in a social field. $J$. Math. Sociol., 19(3):189-219, 1994.

[14] J. Lichtenberg. Democracy and the mass media: a collection of essays. Cambridge University Press, 1990.

[15] G. Toscani. Kinetic models of opinion formation. Commun. Math. Sci., 4(3):481-496, 2006. 
L.B.: Sorbonne Universités, UPMC Univ Paris 06, UMR 7598 LJLL, Paris, F-75005, France \& CNRS, UMR 7598 LJLL, Paris, F-75005, France \& Inria, Équipe-Projet Reo, BP 105, F-78153 Le Chesnay Cedex, France

E-mail address: laurent.boudin@upmc.fr

F.S.: Dipartimento di Matematica, Università degli Studi di Pavia, Via FerRATA 1, I-27100 PAVIA, ItAly

E-mail address: francesco.salvarani@unipv.it 